\title{
Research on Applications of Curvelet Threshold in Seismic Signal Denoising
}

\author{
Hui Yang ${ }^{1}$, Hua Zhang ${ }^{1}$ \\ ${ }^{1}$ East China University of Technology, Nanchang, 330000, China
}

\begin{abstract}
The seismic signal denoising is to extract useful information from seismic data, remove interference noise and improve the signal-noise ratio to lay the foundation of subsequent treatment of the seismic data. The paper gives the basic principles of the curvelet transformation and puts forward the method of reducing the noise in the seismic signal by using the curvelet threshold. Finally, the paper gives a practical example of denoising the seismic signal to provide some references for the relative teachers.
\end{abstract}

Keywords:curvelet threshold, curvelet transformation,seismic signal denoising

\section{Introduction}

The seismic data collected by seismic exploration contains a large amount of noise information, which strongly interferes with the presentation of useful seismic signals to the axis. In order to get the information of the same direction axis, we need to filter the original field seismic information, so as to provide reliable data for the geological interpretation of seismic exploration. The noise of seismic data is mainly some coherent interference wave and random noise. Due to surface conditions, excitation, propagation and reception, coherent noise often appears in seismic records. The existence of coherent noise seriously affects the results of wavelet processing, velocity analysis and correction, and even migration imaging, and the effect of geological interpretation should be eliminated. This paper mainly deals with the processing of the noise signal. Low frequency interference often exists in the original single shot record, especially the surface wave is more serious, because the frequency of the surface wave is generally low, so sometimes using high pass filter method to remove the surface 
wave, but this is not an option because of high pass filtering in eliminate surface wave as well as a complete loss of low frequency composition of effective signal and the low frequency component is to improve the resolution and wave impedance inversion is very important. The two-dimensional filtering, also known as the apparent velocity filtering, it is based on the reflected wave and interference wave propagation speed is different, to remove interference wave prominent effective wave. But in view of the obvious difference of the reflection wave and the surface wave in the frequency, the two-dimensional filter is adopted, and the effect is not obvious. Through the comprehensive analysis of the surface wave, it can be found that there is a big difference between the surface wave and the effective wave, and the surface wave has obvious directivity. Curvelet has a good ability to distinguish directional signals. In this paper, we use the good characteristic of curvelet threshold to achieve the purpose of denoising.

\section{Basic principles of curvelet transformation}

We will introduce the basic principles of the discretecurvelet transformation as the theoretical basis. We take the $\mathrm{f}\left[t_{1}, t_{2}\right], 0 \leq t_{1}, t_{2}<n$ in the Descartes coordinate system. The discretecurvelet transformation is:

$$
C^{D}(\mathrm{j}, \mathrm{l}, \mathrm{k})=\sum_{0 \leq t_{1}, t_{2}<n} \mathrm{f}\left[t_{1}, t_{2}\right] \varphi_{j, l, l}^{D}\left[t_{1}, t_{2}\right]
$$

We take the function of band pass filter:

$$
{ }^{\circ} \mathrm{F}\left(\omega_{1}\right)=\sqrt{{ }^{\circ} \mathrm{F}\left(\omega_{1} / 2\right)^{2}-{ }^{\circ} \mathrm{F}\left(\omega_{1}\right)^{2}}
$$

Now, we definite that:

$$
{ }^{\circ} \mathrm{F}_{j}\left(\omega_{1}\right)={ }^{\circ} \mathrm{F}\left(2^{-j} \omega_{1}\right)
$$

We use the above function to realize the multi-scale segmentation.

For every $\omega=\left(\omega_{1}, \omega_{2}\right), \omega_{1}>0$, we have:

$$
V_{j}\left(S_{\theta} \omega\right)=\mathrm{V}\left(2^{j / 2} \omega_{2} / \omega_{1}-l\right)
$$

In the above formulation, $S_{\theta}$ is a shear matrix. In fact,

$$
S_{\theta}=\left(\begin{array}{cc}
1 & 0 \\
-\tan \theta_{l} & 1
\end{array}\right)
$$

We definite that for every $\theta_{l} \in[-\pi / 4, \pi / 4)$, we have:

$$
U_{j, 1}(\omega)={ }^{\circ} \mathrm{F}\left(\omega_{1}\right) V_{j}\left(S_{\theta} \omega\right) .
$$

The scale and angular depart of the discrete curvelet transformation is shown in Figure 1. 


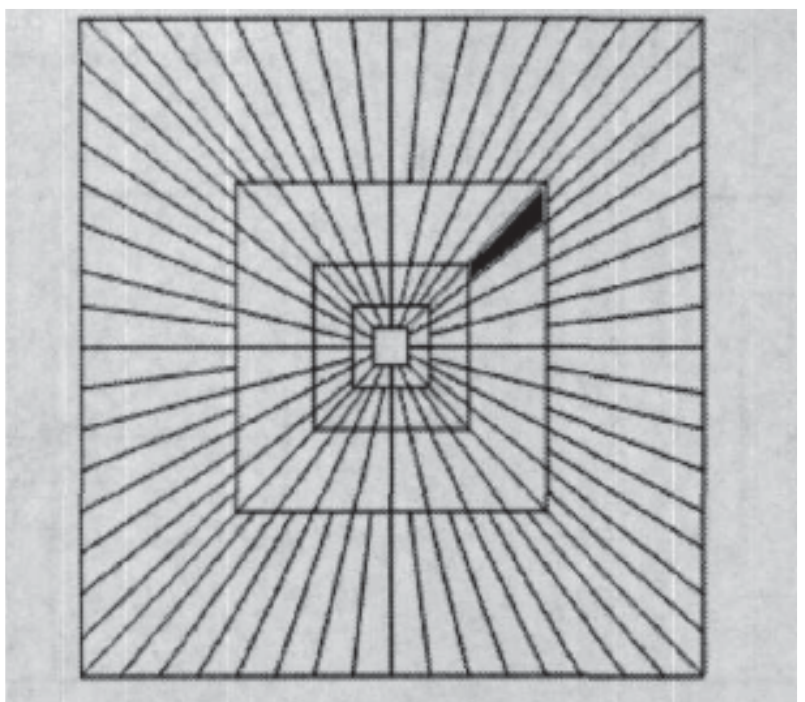

Fig. 1: Scale and angular depart of the discrete curvelet transformation

\section{Applications of curvelet threshold in seismic signal denoising}

Curvelet transformation has a concentrated ability. It can concentrate the main energy of the signal on a small number of curvelet coefficients. The transformation of noise on any orthogonal basis is still noise and has the same magnitude. In contrast, the curvelet series values of the signal are larger than those of the curvelet series with small noise and small energy dispersion. Therefore, the establishment of a suitable threshold function, the curvelet coefficient threshold processing, can achieve the purpose of removing the noise to retain the effective signal. The application of threshold denoising in curvelet domain is a simple and effective method, which is usually used to remove the hard threshold and soft threshold. The hard thresholding method can preserve the local features of the signal, but the pseudophenomenon will appear after denoising. The soft threshold processing is relatively smooth, but it may cause the distortion of signal edge blur. In order to overcome these shortcomings, the use of soft and hard threshold compromise method. When alpha 0 is taken as the hard threshold method, the soft threshold method is used for 1. When the data is required to have a sharp edge, the compromise threshold should be moved to the hard threshold, that is, the value of alpha is between 0.5 and 1 . In order to avoid the pseudo gabs phenomenon, the compromise threshold should be moved to the soft threshold direction, that is, the value of alpha is between 0 and 0.5 . Between 0 and 1 to adjust the size of alpha, you can get better denoising effect. In this paper, a representative alpha of 0.5 is used to remove noise.

Curvelet has the characteristics of linear threshold signal, after curvelet decomposition, the energy is concentrated in the linear range where the curvelet decomposition of the coefficient matrix is. The matrix energy distribution is 
correspondingly greater. Linear signal denoising can make full use of the characteristics of curvelet transformation, related processing by curvelet coefficient of a range, such as the curvelet decomposition of the coefficient matrix in the numerical value of zero or close processing can achieve the signal energy to adjust the curvelet coefficients of the objective. The remaining small part of the noise energy can be used linear noise signal and other signals of different frequency bands, the design of a simple filter to filter out, so as to get a more satisfactory denoising effect. Based on the above denoising theory, a software generated seismic signal velocity model is used to denoise the experiment.

\section{Concrete example}

We chose a district high density single record in a set as a target. High density single shot record without receiving a combination of random noise field, very strong, which increases the difficulty of subsequent data processing. Firstly, we set the diffusion correction, the energy distribution profile uniform, and then the profile of denoising. We should deal with the image overlap subdivision and recombination. It can be seen that using the denoising algorithm can significantly improve the restack data due to the ground noise ratio. The interference source, noise ground micro seismic data will contain some coherence, generally appears in the local channel, a local linear interference. In order to better simulate the ground micro seismic records, the original model is added with a group of strong visual velocity is lower than the effective signal interference noise. In the conventional threshold curvelet transform, the coherent noise energy is strong, the random noise is removed, and the noise in the profile after the threshold denoising of the compensated transform is eliminated. The original seismic signal is shown in Figure 2:

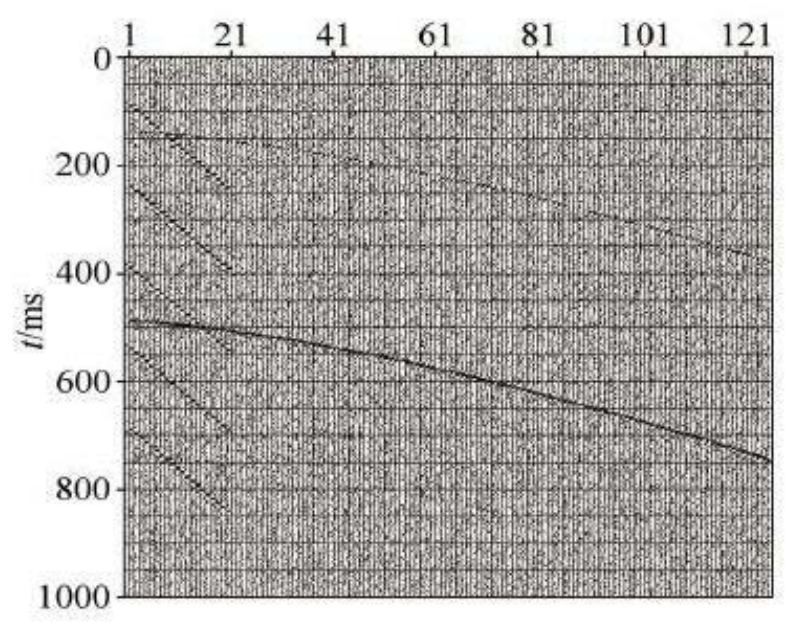

Fig. 2: Figure of original seismic signal 
There is some weak energy part in the original data. The signal-noise ratio is very low. The effective signal energy relative to after dozens of effective signal energy is weak. The continuous back events can be seen as a weak signal. From the denoising post processing results have greatly improved the conventional threshold curvelet transform method, although the random noise profile data contains less, weak signal has been detected, strong energy more continuous phase axis. The conventional threshold curvelet transforms also removes some of the effective signals when the noise is removed. The results show that the compensation threshold curvelet transform is the best, and the phase of the weak signal is continuous. Signal fidelity also further, to better the first break. Curveletdenoising is better than wavelet denoising under the same threshold value. Curveletdenoising has the ability to identify weak in-phase axis, and the linear compression method is the most suitable for weak signal recognition. The threshold value is helpful to keep the weak signal, but the snr of the threshold method is limited. The proposed denoising method can reduce the threshold and keep the weak signal and improve the signal to noise ratio. In this paper, we use the method to reduce the noiseand the fidelity of the profile. In addition, the research shows that this method can extract weak signal. We can see that the image alignments obtained using the algorithm of general breakpoint location is clear, the weak phase axis clear identification, morphological integrity, high fidelity, and the denoising method thoroughly, boundary control section. The experiment shows that the method of reducing the threshold and compensating the small coefficients is effective for weak signal protection and detection. Denoising results are shown in Figure 3:

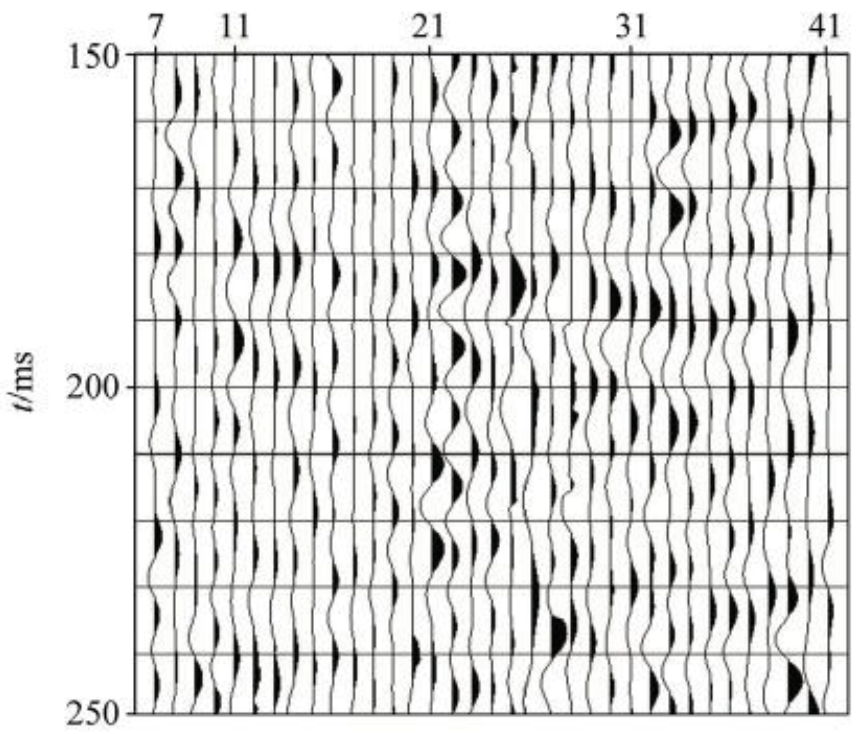

Fig. 3: Seismic signal after denoising 


\section{Conclusion}

There always some interference in the original seismic signal recording. In order to achieve better denoising purposes, thecurvelet threshold can be used to improve the signal-noise ratio. Experiments have verified the feasibility of the method. We believe that the curvelet threshold will play a greater role in the field of seismic signal denoising in the future.

\section{References}

[1]Yang Kai, Liu Wei, Pan Yong, Random Noise Attenuation Based on Soft and HardThreshold Compromise in Curvelet Domain, Chinese Journal of Engineering Geophysics, 10(4), pp. 437-441, 2013.

[2]Liu Lei, Liu Zhen, Zhang Junhua, Denoising and detecting seismic weak signal based on curveletthresholding method, Progress in Geophysics, 26(4), pp. 1415-1422, 2011.

[3]Liu Hao, DuanTianyou, Jiang Tengfei, DuanJiayin, Lu Meiling, Denoising of curvelet transform in micro-seismic signals, Petrochemical Industry Technology, (7), pp. 216, 2015.

[4] He Ke, Zhou Lipin, Yu Baoli, Deng Yong, Wang Lili, Wang Qian, The ground microseismic weak signal detection method based on compensation threshold of curvelet transform, Geophysical and Geochemical Exploration, 40(1), pp. 55-60, 2016. 